\title{
Centenaire du ministère du Travail. Bilan des projets de recherche DARES-CHATEPF
}

\author{
Séminaire du 23 mai 2007
}

A Century of the French Labour Ministry. Conclusions about a research program

\section{OpenEdition \\ Journals}

Édition électronique

URL : http://journals.openedition.org/travailemploi/2220

DOI : 10.4000/travailemploi.2220

ISSN : $1775-416 \mathrm{X}$

Éditeur

DARES - Ministère du Travail

Édition imprimée

Date de publication : 30 décembre 2007

Pagination : 87-104

ISSN : 0224-4365

Référence électronique

"Centenaire du ministère du Travail. Bilan des projets de recherche DARES-CHATEPF », Travail et Emploi [En ligne], 112 | octobre-décembre 2007, mis en ligne le 05 novembre 2010, consulté le 03 mai 2019. URL : http://journals.openedition.org/travailemploi/2220; DOI : 10.4000/travailemploi.2220 


\title{
Centenaire du ministère du Travail. Bilan des projets de recherche DARES-CHATEPF $\left({ }^{*}\right)$
}

\author{
Séminaire du 23 mai 2007
}

En mars 2004, dans le cadre de la préparation du centenaire du ministère du Travail, la Direction de l'animation de la recherche, des études et des statistiques (DARES) et le Comité d'histoire des administrations chargées du travail, de l'emploi et de la formation professionnelle (CHATEFP) ont lancé un appel à projets de recherches sur le thème "Élaborations et mises en ouvre des politiques du Travail: le ministère du Travail et la société française au XXe siècle ». L'objectif du séminaire de recherche qui s'est tenu le 23 mai 2007, et dont nous rendons compte ici, était de tirer le bilan des travaux de recherche financés dans ce cadre et d'en diffuser les conclusions essentielles auprès de l'administration et des chercheurs. La journée était organisée autour de trois tables rondes: le personnel et les structures du ministère du Travail; les acteurs et réseaux du ministère; le ministère du Travail et les organisations professionnelles et syndicales.

\section{Introduction par Michel Lucas, président du CHATEFP}

Cette journée conclut un processus engagé en mars 2004 dans la perspective de la célébration du centenaire du ministère du Travail. Cette commémoration offrait l'occasion aux chercheurs d'engager des travaux sur l'histoire du ministère et l'évolution de ses relations durant cent ans avec d'autres ministères et avec les partenaires sociaux - sujet dont on mesure tous les jours l'actualité. Qui fait quoi dans l'évolution du droit du Travail? Estce que le rôle conventionnel des partenaires sociaux précise ou prolonge l'action du législateur - auquel il reviendrait de définir les objectifs - ou y a-t-il un rôle spécifique et de quelle nature? Existe-t-il une construction conventionnelle parallèlement à celle de l'État? Et concernant ce dernier, sur quel terrain l'État a-t-il évolué au cours de ces cent ans? Y a-til un État local surtout depuis la mise en place des politiques de délocalisation et donc, comment l'État s'est-il déconcentré?

Quarante projets ont été déposés, dix ont été retenus, neuf ont été menés à bonne fin, dont les résultats seront débattus tout au long de la journée autour des trois thèmes suivants: comment appré-

(*) La relecture de la retranscription des débats a été assurée par Odile Join-Lambert, secrétaire scientifique du programme, chercheure en histoire à l'IRES. Les conclusions de la journée, tirées par Odile Join-Lambert et Vincent Viet (DREES), ont servi de trame à la conclusion des numéros 110 et 111 d'avriljuin 2007 et juillet-septembre 2007, que Travail et Emploi a consacrés au centenaire du ministère du Travail (cf. Travail et Emploi $\mathrm{n}^{\circ}$ 111, pp. 75-78). cier le poids relatif du ministère du Travail au cours des cent dernières années, à travers l'étude de son personnel et de ses structures? Quels sont les acteurs et les réseaux concernés? Enfin, comment ont évolué les rôles respectifs de l'État et des acteurs sociaux? Les recherches seront résumées et discutées par un rapporteur, auquel les auteurs des projets répondront en précisant certains points. Un débat sera ensuite ouvert avec, en particulier, l'apport d'importants témoignages.

\section{Table ronde $n^{\circ} 1$ \\ Le personnel et les structures du ministère du Travail \\ Président: Claude Chetcuti \\ Recherches présentées}

Paul-André Rosental, EHESS-CRH, Caroline Douki, université de Paris-VIII, David Feldman, université de Londres, La protection sociale des travailleurs migrants dans l'entre-deux-guerres: le rôle du ministère du Travail dans son environnement national et international (France, Italie, Royaume-Uni).

Béatrice Touchelay, université de Paris-XIIVal-de-Marne, L'émergence des statistiques du travail entre 1891 et 1967 ou la construction d'une réalité économique, politique et sociale.

Étienne Penissat, Laboratoire de sciences sociales de l'ENS, Socio-histoire de la construction des statistiques du ministère du Travail.

Jean-Luc Bodiguel, CNRS-MSH, Ange Guépin, Jean-Pierre Le Crom, CNRS, Laboratoire droit et changement social, L'administration centrale du travail et la production du droit du Travail (1906-1960). 


\section{Discussion par Michel Margairaz, université de Paris-VIII}

Trois questions transversales aux quatre rapports peuvent être posées. La première question concerne la façon dont on apprécie le poids relatif du ministère du Travail, qu'il s'agisse de la construction des statistiques du Travail, de celle du droit social ou la politique migratoire: que se donne-t-on comme instrument de mesure pour saisir le poids relatif du ministère par rapport aux autres acteurs publics et privés? Il serait bon que les chercheurs l'explicitent mieux. À l'intérieur du ministère du Travail, quels sont les bureaux ou les directions qui pèsent? Et quel est le rôle relatif du ministre? Y a-t-il des ministres forts et des ministres faibles? Quelle est la marge de manœuvre de ces derniers par rapport aux structures? Prenons l'exemple paradoxal de 1936: le ministre du Travail Jean-Baptiste Lebas est particulièrement absent dans une actualité où le travail jouait un rôle important et où il aurait été logique qu'il soit présent lors de l'accord Matignon, alors que c'est Roger Salengro, le ministre de l'Intérieur, qui est présent.

La deuxième question transversale concerne la pondération entre l'histoire internaliste des structures et des personnels du ministère et l'histoire externe des autres acteurs. Selon les cas, cette question ne peut pas être traitée de la même manière et bien entendu, lorsqu'il s'agit d'étudier le travail des bureaux et des pratiques administratives, l'histoire est délibérément internaliste. Mais sur l'usage des statistiques ou en matière migratoire, il y a évidemment à combiner une histoire interne et une histoire externe. Trois types de configurations externes pèsent sur l'activité du ministère du Travail. D'abord, une configuration gouvernementale et institutionnelle, présente dans les recherches mais pas toujours de manière suffisamment explicite. Dans les rapports de la France avec l'Italie et la Grande-Bretagne sur la politique migratoire notamment, jusqu'à quel point le ministère du Travail est-il un ministère faible par rapport à des ministères forts comme les Finances, l'Intérieur, les Affaires étrangères? Étant entendu que même un ministère faible peut avoir la force du faible qui réside, notamment, dans la division des forts. Lorsque des rivalités importantes existent entre ministères, il y a une marge de manœuvre pour un ministère faible. Il existe une deuxième configuration externe, sociopolitique cette fois. Le ministère du Travail est ouvert sur les acteurs sociaux, les syndicats, les organisations professionnelles. Il est aussi en liaison avec eux pour définir tout ce qui concerne la législation sociale et la réglementation. Les recherches mettent en général en perspective leur poids effectif variable, selon la conjoncture. Une dernière configuration, intellectuelle (les outils d'analyse, de perception, de conceptualisation) aurait pu être davantage développée: quels sont les relais, les modes de transmission du débat scientifique et intellectuel hors du ministère et à l'intérieur? Comment le ministère lui-même s'insinue dans un débat scientifique et intellectuel sur des questions qui, par ailleurs animent une autre scène qui n'est pas la scène administrative mais qui est, effectivement, la scène du débat scientifique et intellectuel? Une dernière question transversale aux quatre recherches concerne la périodisation: peuton arriver avec l'ensemble des recherches de cette table ronde, qui traversent les cent ans d'histoire du ministère, à établir des temps forts ou faibles?

\section{La protection sociale des travailleurs migrants dans l'entre-deux-guerres}

\section{- Michel Margairaz}

Le rapport de recherche sur la protection sociale des travailleurs migrants est une étude croisée et comparée de la France, de l'Italie et du RoyaumeUni sur le rôle du ministère du Travail dans son environnement national et international concernant la protection des travailleurs migrants entre les deux guerres. Les auteurs étudient le poids relatif du ministère du Travail parmi les autres acteurs nationaux et internationaux. Le grand intérêt de cette recherche réside dans ces attendus scientifiques, problématiques et théoriques: les trois auteurs montrent que l'idée d'une tyrannie $\mathrm{du}$ «national» doit être fortement relativisée par le rôle des organismes privés et du patronat, notamment. Les auteurs étudient le rôle joué par le droit international entre les deux guerres, auquel contribuent le BIT et une «nébuleuse réformatrice» internationale. Les pays ont des intérêts différents (entre pays d'immigration et d'émigration) et en leur sein les différents acteurs publics également (les Affaires étrangères sont plus volontiers ouvertes à la circulation tandis que le ministère du Travail ou celui de l'Intérieur peuvent davantage mener une politique de régulation, dans des sens qui peuvent être eux-mêmes différents d'ailleurs). Deux questions se posent donc. Dans les trois pays, le ministère du Travail pèse assez peu et il serait vraiment intéressant de savoir comment se décline le «assez peu». Une deuxième question porte sur le contexte des années 1930 (ce qui est surtout développé concerne les années 1920), où une autre circulation internationale se met en place, les pays se refermant sur eux-mêmes aux dépens du BIT.

\section{- Claude Chetcuti, inspecteur général des affaires sociales honoraire, président de séance}

Il serait intéressant de savoir ce qu'est le ministère du Travail en 1906, entre les deux guerres, et en 1940. Pour mieux l'expliquer, il faudrait déterminer les rôles respectifs du ministère du Travail et du ministère des Finances, qui se substitue à lui et le marginalise. Par exemple, le contrôle général des 
assurances était-il un outil qu'on pouvait utiliser? En tout cas, la Direction des assurances devait être certainement utilisée, mais elle n'est rattachée aux Finances qu'en octobre 1940. Le fait qu'un champ social soit unifié sous l'égide d'un seul ministre avec une vocation sociétale comme en 1906, comme aujourd'hui avec le ministère de Monsieur Borloo montre bien que la configuration et donc le «poids » du ministère peuvent se transformer.

\section{- Paul-André Rosental}

Nous avons centré notre travail sur les années 1920 parce que c'est une période qui nous a semblé, à l'issue de la recherche, assez matricielle. Sur les années 1930, nous prolongerons notre enquête, ce qui nous permettra de mesurer l'effet de l'ensemble des constructions internationales sur la situation des migrants. On peut déjà observer pour cette période une différence de sort selon les nationalités de migrants.

Le ministère du Travail joue, à bien des égards, un rôle spécifique. D'abord, parce qu'il contribue à l'équilibre ou au conflit interministériel en fonction des évolutions internes du marché de l'emploi et des rapports avec les syndicats. En deuxième lieu, parce qu'il administre en partenariat avec les entreprises et en particulier avec les entreprises de migration, la mise en place et la gestion des filières migratoires en favorisant, notamment, le tropisme bien connu en faveur des migrants d'Europe centrale et d'Europe orientale. Dans l'administration des filières migratoires d'approvisionnement de la main-d'œuvre, la France, via le ministère du Travail, met en place des antennes de recrutement, en Europe centrale et orientale. L'intérêt pour l'historien de l'étude de ces antennes, c'est qu'elles sont mixtes. C'est-à-dire qu'il y a une très large délégation aux entreprises. Il y a deux types d'entreprises: de main-d'œuvre et de migration. Ce partenariat donne un poids très important aux entreprises. La question qui se pose classiquement à l'histoire de la migration est l'existence de ces filières préférentielles. Parmi les fonctions qu'a pu avoir le ministère du Travail, l'une d'entre elles est de les officialiser, même s'il a de faibles moyens pour cela. En troisième lieu, le ministère du Travail représente la France au BIT. Il bénéficie, par là même, d'un levier pour essayer de défendre sur le plan diplomatique ses intérêts et ceux des lobbies avec lesquels il est en interaction. Vis-à-vis des syndicats, il s'agit pour lui de mettre à niveau les conditions sociales des travailleurs migrants avec celles des nationaux. En ce qui concerne les employeurs, il s'agit d'essayer de faire en sorte que le coût du travail à l'étranger soit comparable au coût du travail en France et également de faire en sorte qu'il y ait un contrôle de la main-d'œuvre que les entreprises font acheminer (autrement dit pas de départ immédiat des ouvriers acheminés de l'étranger).

\section{- Caroline Douki}

Dans le cas de l'Italie, il s'agit d'analyser l'un des interlocuteurs principaux des gouvernements et du ministère français du Travail dans l'élaboration d'une politique migratoire. L'Italie est à partir la fin du $\mathrm{XIX}^{\mathrm{e}}$ siècle l'un des pays d'émigration les plus importants au monde, et notamment le principal pays émetteur à destination de la France. Depuis le tout début du $\mathrm{XX}^{\mathrm{e}}$ siècle, l'État italien a une politique volontariste de négociations bilatérales avec tous les pays qui reçoivent ces émigrants. En fait, pour le ministère du Travail français, l'interlocuteur italien est tout à fait composite. Plusieurs interlocuteurs interviennent et font pression dans les négociations bilatérales ou dans les ententes internationales: le ministère des Affaires étrangères italien, le ministère de l'Agriculture, de l'Industrie et du Commerce italien, le ministère du Travail quand il existe et, enfin, une configuration interministérielle, le Commissariat général à l'immigration, qui rassemble les intérêts les plus composites des ministères italiens.

\section{Les statistiques du Travail}

\section{- Michel Margairaz}

Quelle est la place occupée par les cadres conceptuels, techniques, et administratifs dans l'élaboration des fonctions statistiques au sein du ministère du Travail? Pour y répondre, la recherche de Béatrice Touchelay comporte plusieurs intérêts majeurs. D'abord, elle montre bien comment 1'élaboration des statistiques se trouve au cœur de rivalités très importantes sur le terrain administratif. Même si administrativement, la Statistique générale de la France (SGF) est rattachée jusqu'en 1934 au ministère du Travail, il s'agit d'un monde à part avec ses logiques propres. Peut-on identifier facilement la SGF au ministère du Travail en tant que tel? Ne faut-il pas aller voir de près plutôt en quoi la SGF utilise les données fournies par le ministère? On voit bien comment la statistique s'émancipe du ministère du Travail pour être rattachée progressivement à partir de 1934 aux Finances et rejoindre le domaine économique et financier. La preuve statistique du ministère du Travail devient de plus en plus économique. Une autre source d'étonnement vient de ce que la fonction statistique à l'intérieur du ministère recule paradoxalement, dès la création du ministère en 1906, alors qu'elle avait un rôle plus important avant la création du ministère avec l'Office du travail. Il faut attendre l'après Seconde Guerre mondiale pour que la fonction statistique s'autonomise à l'intérieur du ministère, l'enquête ACEMO en étant une bonne illustration. La question des rapports entre les statistiques du Travail et la législation sociale mérite, en tout cas, d'être étudiée au cas par cas et dans des conjonctures très particulières. Béatrice Touchelay montre qu'il n'y a pas de parallélisme entre la production 
de statistiques relatives au travail et l'ampleur de la législation sociale. Il n'existe pas de vision théorique normative selon laquelle on ferait de bonnes lois sociales parce qu'on aurait fait un bon travail statistique préalable. C'est plutôt la législation sociale qui constitue un aiguillon au cours de la production statistique pour accompagner l'application des lois sociales. Les deux histoires semblent donc relativement découplées.

Une troisième question porte sur le rôle des statistiques dans la décision politique. Il n'y a pas parallélisme entre les décisions politiques relatives aux questions du travail et l'élaboration statistique préalable. La statistique vient toujours après les décisions politiques dans le cadre de l'histoire sociale française. Jusqu'à quel point le ministère est-il dépendant d'autres forces sociales pour l'élaboration de ses propres statistiques, notamment à l'égard des données issues du monde patronal et syndical en général? Ce n'est pas spécifique au ministère du Travail, on retrouve le même problème pour le Conseil national économique, à la SGF, dans les services statistiques à l'intérieur des Finances, etc. Une quatrième série de questions porte sur la périodisation. Les deux guerres mondiales sont, pour des raisons différentes, des périodes de maturation de pratiques pour le ministère du Travail, pratiques qui d'ailleurs tournent court pour un certain nombre d'entre elles: autrement dit, est-ce que les deux guerres sont des «parenthèses »? Pendant la Première Guerre mondiale, quel est le rôle d'Albert Thomas notamment? Après la Seconde Guerre mondiale, la politique des revenus échoue mais elle permet d'autres choses qu'on aimerait mieux connaître.

Enfin, et cette question concerne plus particulièrement la recherche d'Étienne Pénissat, la vision plus attentive à la conjoncture et aux fluctuations qui se développe dans l'entre-deux-guerres est-elle liée à des débats scientifiques? Une personnalité comme Simiand joue-t-elle un rôle? Ne faut-il pas rapprocher cette vision de celle qui va se retrouver à la SGF puis à l'Institut de conjonctures? Par ailleurs, n'a-t-on pas perdu dans ce domaine une vision qualitative intéressante de ce que pouvaient être les salaires (les salaires dans leur configuration à la fois locale et différenciée selon les types de production, de produit, de marchés, etc. )? Ne faudrait-il pas mieux mettre en évidence une impasse: d'une part, on n'arrive pas à faire remonter des informations suffisantes et d'autre part, le ministère du Travail est tributaire des sources patronales et n'arrive pas, tant qu'il n'y a pas d'obligation, à obtenir que les questionnaires soient remplis. Est-ce que cette obligation qui arrive en 1938 constitue une «révolution culturelle»?

\section{- Claude Chetcuti}

Ce que le ministère de la Main-d'CEuvre, l'INSEE ou la DARES entendent par «statisti- ques» est assez différent de ce qu'entendaient les premier, deuxième et cinquième bureaux en 1938 ou même la Direction générale du travail et de l'emploi dans la période 1945-1962. On a tendance à surévaluer le rôle le rôle d'information des inspecteurs du Travail. Peut-on penser que l'inspecteur était mieux formé à la statistique grâce à des circulaires ministérielles? J'en doute. Qu'est-ce que les inspecteurs ont vu et dans quelles proportions? Tous les rapports montrent qu'ils sont dans l'incapacité, vu leur nombre, de visiter toutes les entreprises même les plus importantes. Le problème me paraît le même pour ce qui est de l'enquête auprès des conseils de prud'hommes: que représentent les prud'hommes comme champ de couverture géographique en dehors des grandes villes ou de quelques pôles industriels? Ma dernière question porte sur les problèmes de sources. J'ai travaillé sur les archives départementales du Nord et du Pas-de-Calais. Les archives locales contiennent un certain nombre d'instructions envoyées par Paris, mais en revanche il n'y figure jamais les remontées. On sait que les inspecteurs du Travail sont censés donner tel ou tel renseignement, mais on ne connaît pas, jusqu'à la fin des années 1970, les suites concrètes données à leurs observations.

\section{- Étienne Pénissat}

Au début des années 1920 un travail est fait par les proches de la Société statistique de Paris et notamment par Simiand qui y est fortement investi. Il s'agit d'un travail de lobbing auprès du ministère du Travail et auprès de la présidence du Conseil pour que des enquêtes statistiques plus fréquentes se développent et jouent un rôle de relais. Il fait entrer, par exemple, plusieurs agents du ministère du Travail à la Société statistique de Paris, notamment C. Piquenard, ancien directeur du Travail. $\mathrm{Au}$ BIT aussi, il existe une conférence des statisticiens du Travail, réunissant presque tous les deux ans des membres de la SGF et du ministère du Travail. Ils tentent d'harmoniser les statistiques, notamment en matière de salaire et de chômage. Si ce travail d'harmonisation et de développement d'enquêtes proprement statistiques est souvent repoussé par interventions des représentants patronaux, par contre on note, dans les publications du ministère un effort important de rationalisation (lisibilité du rubricage, usage de tableaux et de graphiques) et de comparaison des séries statistiques entre elles (par exemple comparaison entre les chômeurs secourus et les chômeurs inscrits dans les bureaux de placement ou encore comparaison des séries de chômeurs avec des séries concernant l'évolution de la production industrielle). Preuve qu'une culture statistique se développe même si elle reste mineure au sein de ce ministère. Pour terminer sur la question de la conjoncture, elle joue un rôle particulier au début des années 1930 avec l'enquête sur les effectifs des établissements de plus de cent salariés. Elle devient mensuelle et est fondée sur les remontées des 
inspecteurs du Travail. Sur la fiabilité de ces remontées, dans les archives que j'ai consultées, beaucoup d'inspecteurs du Travail refusent de faire remonter des chiffres à la direction du Travail au motif qu'ils ne peuvent pas faire le contrôle et en même temps assurer une remontée d'informations, puisqu'ils n'ont pas accès aux livres de payes, par exemple, en dehors des cas d'infractions. L'alternative consiste alors à passer par les conseils de prud'hommes ou par les salaires indiqués dans les conventions collectives. Si bien que le patronat et les syndicats produisent eux-mêmes leurs propres chiffres.

\section{- Béatrice Touchelay}

Étudier les salaires «administrés» pendant la Seconde Guerre mondiale avec le STO supposerait un travail en soi. En ce qui concerne le rôle des inspecteurs du Travail dans les enquêtes ACEMO, cela marche mieux à partir de 1951 car précisément les inspecteurs n'interviennent plus dans les enquêtes: les fonctions de contrôle et de collecte des informations ne peuvent plus être confondues. C'est justement dans ces années 1950 qu'est créé un service de statistiques au ministère. Ce service de statistiques du ministère du Travail avait une autonomie et pouvait produire des statistiques à partir d'informations qu'il allait collecter lui-même. L'étude des années 1955-1960 mériterait certainement d'être approfondie: à mon avis, la différence essentielle réside entre l'avant et l'après fin des années cinquante où il y a désormais une demande politique de statistiques. Avant, c'est le patronat qui demande le maintien des commissions départementales du coût de la vie, alors qu'elles produisent des statistiques erronées. Tout simplement parce qu'il veut savoir à partir de quand il doit négocier ou accepter une augmentation, comme j'ai pu le constater en consultant les archives départementales de Lyon.

\section{- Michel Margairaz}

L'exactitude des chiffres importe peu en réalité. C'est leur comparaison par rapport aux chiffres de l'année précédente qui compte. Le problème n'est pas de connaître le coût de la vie d'un point de vue scientifique, mais de savoir s'il a augmenté ou non par rapport à l'année précédente: n'est-ce pas cela le tournant conjoncturel, dans la mesure où on est dans des périodes de baisse puis de hausse des prix? Comme les fluctuations ont été très brutales dans les années 1930, il faut avoir un outil de repérage par rapport à l'avant. Là réside un des éléments d'explication qui n'est pas une question de véracité ou d'exactitude du chiffre mais d'adaptation aux besoins des utilisateurs.

\section{- Claude Chetcuti}

On peut se demander si ce n'est pas en sens inverse que cela fonctionne. Autrement dit, avec les conventions collectives, c'est le syndicat patronal qui élabore la grille de salaires, si on suit l'exemple du syndicat patronal textile du Nord. La situation est variable selon les départements, suivant la force ou la faiblesse des organisations syndicales, suivant aussi le nombre et l'implantation des inspecteurs du Travail (en 1933, dans la moitié ouest de la France, en dehors des très grandes villes, il n'y a même pas un inspecteur par département).

\section{- Vincent Viet (MIRE - DRESS)}

La protection sociale donne lieu à une production statistique importante et on aimerait en savoir plus sur cette question, ne serait-ce que pour nuancer l'idée selon laquelle le ministère du Travail est incapable de produire des statistiques propres. Il s'agit, en tout cas, de statistiques qui ne sont pas d'origine patronale et qui portent sur le fonctionnement du système de protection sociale français.

\section{- Claude Chetcuti}

On touche au fonctionnement même du ministère organisé à partir des années 1930 en deux grandes entités : la Direction générale des assurances sociales (après 1945, Direction générale de la Sécurité sociale) et la Direction du travail (puis Direction générale du travail et de la main-d'œuvre, dont les branches auront suivant les moments une autonomie plus ou moins grande, voire totale). Dans un cas, on a affaire à une administration qui fabrique une réglementation et s'efforce de savoir si elle est appliquée par des enquêtes spécifiques; dans l'autre cas, la Direction des assurances sociales est, dès le départ, conçue comme un organisme de tutelle, mais aussi de gestion du régime à travers le réseau des caisses départementales. La Direction du travail collecte des informations sur les salaires mais ne gère pas le flux, tandis qu'en ce qui concerne les assurances sociales, l'information ne compte que pour éclairer la gestion (combien de gens sont couverts, immatriculés, cotisent durant les douze mois de l'année, etc.).

Lors de la création du ministère des Affaires sociales en 1966, le nouveau ministre J.-M. Jeanneney veut une grande direction de la Prévision et des Statistiques. Cela correspond à une demande politique, qui est certaine, mais qu'en est-il de la demande administrative, celle des deux directions générales? En ce qui concerne la Direction du travail et celle de la maind'œuvre, on peut penser qu'elle était faible, pour ne pas dire nulle, avant leur réunification en 1956 et surtout l'arrivée de P. Laurent l'année suivante.

\section{- Témoignage de Raymond Lévy-Bruhl}

J'ai été pendant les années 1950 le chef de la division statistique du ministère du Travail et j'aimerais répondre à quelques-unes des questions, dans la mesure où mes souvenirs me le permettent. La première concerne les rapports avec les différentes parties prenantes de l'administration. Je crois que le ministre du Travail dans les années 1950 avait une 
position un peu intermédiaire, peut-être meilleure qu'au début du siècle mais moins bonne que dans les années plus récentes. En revanche, le ministre du Travail, pendant les années 1950, a été un patron extrêmement agréable et solide pour la statistique. Aussi bien Paul Bacon qu'Albert Gazier étaient des hommes qui pensaient que l'information pouvait être à la fois fiable et désagréable. Elle était ce qu'elle était. J'ai trouvé dans ces deux ministres une aide extrêmement importante pour le développement du service statistique dont j'avais la responsabilité. De même de la part de Pierre Laurent. En revanche, l'administration du ministère du Travail était restée un peu sclérosée sur le plan de la statistique; sans être hostile, elle avait une position très neutre et ne voyait pas l'intérêt des travaux que nous faisions.

Je voulais simplement développer les raisons pour lesquelles la division statistique a pu s'imposer dans ce ministère qui n'y était pas trop favorable. D'abord, c'est qu'elle n'a été que statistique. Elle n'est devenue économique qu'avec la création du SES, puis de la DARES. Ensuite, la division statistique avait une position un peu autonome, à cheval sur le Travail d'une part, la Main-d'Euvre d'autre part. Elle était dirigée par un administrateur de l'INSEE qui ne dépendait pas des structures du ministère. Cela n'empêchait pas l'avancement des administrateurs des différents bureaux. D'autre part, cette division statistique avait été munie d'un atelier mécanographique relativement important pour l'époque, suffisant pour les travaux de la statistique et lui permettant une indépendance concernant l'exploitation. Il était doté de machines tabulatrices permettant de faire l'exploitation de l'enquête ACEMO dans des délais très rapides. Il jouissait d'une certaine indépendance dans la mesure où les documents lui parvenaient directement. Il exploitait et publiait directement sans que personne ne puisse intervenir dans cette chaîne de production des statistiques. Un dernier élément dont personne n'a parlé: à cette époque les travaux internationaux ont commencé, dans le domaine statistique, à se développer beaucoup plus. Il y avait effectivement le BIT mais du fait de la création de la CECA et de l'obligation pour elle d'obtenir des données de bonnes qualités, une activité internationale importante s'est développée à Luxembourg. Cette activité s'est poursuivie ensuite dans le cadre de la CEE mais elle a débuté avant. Elle a été un aiguillon pour faire comprendre au patronat d'une part, aux syndicats ouvriers d'autre part, l'importance du développement de la statistique et de la concertation entre les États. Je signalerai enfin qu'il y avait une organisation dont on parle très peu, l'Union de l'Europe occidentale (UEO) qui, sur le plan statistique, avait un comité qui se réunissait souvent, soucieux de mieux connaître ce qui se faisait dans les autres pays.

\section{- Témoignage d'Annie Fouquet, inspecteur général des Affaires sociales}

Je ne parlerai que de choses que j'ai vécues. Je suis une contractuelle, et c'est en arrivant au ministère du Travail, après dix-neuf années passées à l'Insee, que j'ai compris toute la différence entre le statut de contractuel et celui de fonctionnaire: je n'avais pas la signature. C'était la première fois que le fait d'être contractuelle m'empêchait de faire quelque chose. J'ai découvert également le ministère du Travail, l'importance des acteurs sociaux, notamment des syndicats, dans le travail même du ministère. Si j'avais choisi de venir à l'INSEE à la fin de mes études, c'était bien avec l'idée que la statistique devait servir le débat public.

J'ai d'abord dirigé la division des salaires et conventions salariales, puis j'ai succédé à Mireille Elbaum en tant qu'adjointe au chef du service statistiques et j'ai rempli après son départ les fonctions de chef par intérim pour la transformation du SES en DARES. Quand je suis arrivée, j'ai été reçue par le directeur adjoint du cabinet de l'époque de Jean-Pierre Soisson, Jean Marimbert. Il m'a dit pour m'accueillir: «Faites de bonnes études, nous ferons de bonnes politiques». J'étais partie toute ragaillardie. La création de la DARES a été une décision de la ministre de l'époque, Martine Aubry, qui s'est trouvée confrontée au problème suivant. Depuis le milieu des années 1980, les crédits consacrés à la politique de l'emploi allaient croissants et la négociation avec Bercy devenait de plus en plus difficile. Bercy pouvait chaque année, dans la négociation budgétaire, s'appuyer sur les troupes importantes de son bureau emploi-salaire, qui étaient capables de prévoir l'impact économique des crédits dédiés à la politique de l'emploi sur l'ensemble de l'économie française, dans une perspective à la fois micro et macro-économique. Le rapport de forces était défavorable au ministère du Travail, qui n'avait pas les outils permettant de mesurer l'importance et l'impact attendu des crédits des politiques de l'emploi. Il manquait au ministère une fonction d'analyse macro-économique et de prospective... À l'époque, c'était au sein de la Délégation à l'emploi que quelques contractuels brillants, économistes, ayant quitté le SES pour faire une mobilité auprès du délégué à l'emploi, faisaient dans la mission synthèse quelques calculs de coins de table avec une calculette et surtout beaucoup de règles de 3 .

Il existait néanmoins un précédent très positif au ministère de l'Éducation, qui avait créé une Direction de l'évaluation et de la prospective. Cette Direction avait conféré au ministère de l'Éducation une très grande visibilité, notamment sous l'impulsion de Claude Thélot, qui savait sortir les statistiques du ministère de l'Éducation et avait acquis une grande aura publique sur l'évaluation des lycées, des collèges, etc. C'est une direction semblable que Martine Aubry a souhaité mettre en place. Elle a de- 
mandé pour cela un rapport à Gabriel Mignot, qui a fait une analyse de la fonction études, statistiques et prospectives nécessaires, à l'époque, au ministère du Travail, notamment en regroupant les statistiques du travail, celles de l'emploi, mais également de la formation professionnelle, sorte d'électron libre, dont les statistiques avaient été confiées au CEREQ créé en 1971.

Pour terminer, je voudrais simplement faire un retour sur le passé du travail sur la statistique, qui commence à l'Office du travail créé en 1891. Tout le débat social qui a précédé la création de l'Office du travail - et qu'on a évoqué à l'occasion de son centenaire en 1991 - s'est reproduit un siècle plus tard. J'étais à l'époque au service statistiques: Robert Salais avait organisé au Sénat un colloque à partir des travaux historiques portant sur la création de l'Office du travail, publiés sous la direction de Jean Luciani. Ce qui a été très frappant dans tous ces débats, c'est le souci d'objectiver les relations entre le travail et le capital. La volonté d'observer et de connaître le social est à l'origine de la fonction statisticienne du ministère du Travail, même si, dans les années 1920-1930 cette volonté s'est émoussée. Il faut «substituer au jugement de valeur un jugement des réalités»: c'est encore ce que doit faire la DARES aujourd'hui ou la statistique en général. L'apport de la DARES avant mon arrivée en 2000 a été, sous la responsabilité et l'action remarquable de Claude Seibel, de mettre en place cette fonction de prospective et cette fonction d'évaluation des politiques. On sait bien que les statistiques recouvrent plusieurs choses : le suivi de l'activité, ce qu'on appellerait aujourd'hui le contrôle de gestion; les réalisations; éventuellement, les résultats : est-ce que ce qu'on a voulu faire a bien été fait, notamment dans l'application de la législation? Mais aussi les effets globaux sur l'ensemble des équilibres sociaux et notamment sur la situation sociale? On retrouve bien là ce qui avait été le projet initial de l'Office du travail, lui-même, à la création du ministère du Travail.

\section{L’administration du Travail et la fabrication du droit}

\section{- Michel Margairaz}

Le rapport de Jean-Pierre Le Crom et Jean-Luc Bodiguel sur la fabrication du droit présente une vision interne tout à fait intéressante. L'objectif est de faire l'histoire du travail concret des bureaux du ministère Travail dans l'élaboration du droit social. Sont étudiés les promotions, le rôle des femmes, celui des administrateurs civils, le poids important de certaines personnalités ou directions et l'organisation des structures du ministère par bureaux: le deuxième bureau, le cinquième bureau, le travail documentaire, le travail rédactionnel, le rôle de la Revue française du travail, de la rénovation après 1945. On a envie d'en savoir plus sur les éléments de conjoncture et notamment la période de Vichy. De même, il serait utile d'avoir un exemple précis du type de travail fait sur les textes.

\section{- Claude Chetcuti}

Il serait intéressant de distinguer la Direction du travail du ministère, notamment dans la recherche sur la fabrication du droit du travail. En fait, sur cent vingt administrateurs civils ou rédacteurs dans les années 1930, il y en a une cinquantaine ou une quarantaine à la Direction du travail. Les autres sont à l'Assurance sociale, à la Mutualité, etc. Est-ce que les administrateurs civils et les rédacteurs ont le même profil? Font-ils le même travail? Ont-ils le même poids suivant qu'ils sont dans telle ou telle direction?

\section{- Jean-Pierre Le Crom}

La question du juridisme du ministère du Travail se pose de manière différente selon les époques mais dès le lendemain de la Deuxième Guerre mondiale, il était déjà remis en cause. On peut identifier deux groupes différents au sein du ministère du Travail: les anciens qui sont dans la tradition protectrice du ministère du Travail et les nouveaux qui sont souvent des contractuels. Dans quelle mesure cette différentiation est-elle construite par les chercheurs? Pierre Fournier, dans son témoignage Quarante ans place de Fontenoy évoque assez largement cette question, mais il a aussi lu Michel Crozier et Jacques Lautman qui ont travaillé sur le ministère du Travail... En ce qui concerne la période de Vichy, la question des salaires a été traitée par Bob Handke et moi-même. Les salaires à partir de 1943 constituent un élément du débat franco-allemand sur la main-d'œuvre. À partir du moment où les Allemands s'aperçoivent que le STO ne marche pas très bien, ils acceptent que le gouvernement français fasse des augmentations de salaires, mais qui sont négociés branche par branche avec les Allemands en fonction de l'utilité desdites branches pour l'économie de guerre allemande. Sous Vichy, du point de vue de l'administration et de la production des normes, deux choses sont très importantes. La première, c'est que la nouvelle Direction de l'organisation sociale est censée mettre en œuvre la charte du travail. L'administration est totalement absente, cela se passe entre R. Belin et ses collaborateurs directs et les proches traditionalistes du maréchal Pétain. Deuxièmement, ce qui domine est la question de main-d'œuvre: est-ce que c'est le ministère du Travail qui doit avoir la responsabilité des questions de main-d'œuvre ou est-ce qu'il faut que ce soit une responsabilité interministérielle parce qu'on crée des services nouveaux?

\section{- Claude Chetcuti}

L'étude du personnel constitue en effet un sujet central pour comprendre comment se construit le droit du Travail. Ce qui ressort, c'est la présence d'un personnel de qualité moyenne avec quelques 
brillantes exceptions, ce qui n'est pas spécifique au ministère du Travail et justifie les réformes de 1945 . En revanche, ce n'est pas parce que sont présents de brillants esprits qu'ils vont participer concrètement à l'élaboration du droit du Travail ou à l'élaboration de politiques publiques. Les administrations centrales ont des dossiers et font des études avec un personnel «moyen» qui permet de faire ressortir un jour ou l'autre des choses intéressantes et originales. Peut-on plus précisément voir s'il y a des différences ou non dans la filière de progression entre la direction du travail et par exemple la Direction des assurances sociales? À la Direction générale du travail et de l'emploi dans les années 1960, les contractuels apparaissent en nombre important. Certains conseillent directement le directeur général et jouent un rôle central dans la définition et la mise en œuvre des politiques de l'emploi (Yves Chaigneau, notamment). Les bureaux traditionnels sont court-circuités. Quand on veut innover avec la création du Fonds national de l'emploi, on prend des gens différents de ceux d'alors; les politiques nouvelles sont faites par des personnels différents dans des structures différentes.

\section{- Jean-Luc Bodiguel}

Nous nous sommes limités à la période d'avant 1960, mais il faudrait certainement approfondir la période d'après, où seule une minorité des fonctionnaires passe d'une direction à l'autre. C'est à la direction du Travail qu'ils ont été les plus mobiles, et à celle des Assurances sociales qu'ils ont été les plus immobiles. Je l'explique par la technicité nécessaire pour travailler dans cette direction, en particulier pour les vérificateurs qui devaient avoir des connaissances mathématiques très pointues. Avant 1960, je n'ai pas vu apparaître d'autres profils de fonctionnaires dans la fabrication du droit du Travail; après, les choses changent de manière significative.

\section{- Témoignage de Daniel Lejeune, inspecteur général des Affaires Sociales}

J'interviendrai avec une casquette d'ancien de la direction des relations du Travail à propos de la recherche sur le rôle de l'administration du Travail dans l'élaboration des textes et la fabrication du droit du Travail. J'ai bien vu que l'approche développée par cette recherche était internaliste, ce qui pose un véritable problème parce que je ne suis pas sûr qu'on puisse traiter cette question en se limitant à cette perspective, si du moins je me réfère à ma propre expérience. Certes, je n'ai pas connu les années antérieures à 1960, mais j'ai constaté, pour la période suivante, que la "nébuleuse réformatrice» comportait l'administration, des syndicalistes, des universitaires auxquels il faudrait rajouter, dans certains domaines, d'autres institutions, comme les caisses d'assurances maladie, etc. Je crois que cette nébuleuse a survécu à la guerre de 1914 et qu'elle continue d'être un élément fondamental dans l'élaboration des textes. De sorte que ce qui est permanent, ce ne sont pas tant les fonctionnaires mais un certain nombre de concepts. En tout cas, dans le domaine que je connais bien, celui de la prévention des risques professionnels, ce qui a été permanent depuis la Libération, c'est un certain nombre de concepts qui ont pu évoluer, qui ont pu se succéder, mais qui ont été générés par cette nébuleuse. Au sein de cette nébuleuse, l'administration joue, effectivement, un certain rôle de pivot et elle travaille en permanence sur la base de ces concepts qui sont la matière fondamentale de son action, tels que celui de protection collective, de sécurité intégrée, d'évaluation des risques ou d'identification et de prévention des risques différés. Ces concepts apparaissent parfois sous la plume de l'un ou l'autre, mais ils sont élaborés collectivement par cette nébuleuse.

Et dans ce cadre-là, le rôle de l'administration repose sur un dialogue important, riche, en tout cas dans le domaine de la prévention des risques professionnels, avec les organisations syndicales qui ne sont pas seulement porteuses de revendications mais qui contribuent, très concrètement, à l'élaboration des textes et qui les infléchissent. Le Parlement est moins présent, mais cela dépend des matières. Cette distinction entre le politique et le technique est plus complexe qu'en apparence. Bien sûr il existe des matières plus pointues, comme la prévention des risques professionnels, mais elles n'en sont pas moins politiques, même si c'est moins ressenti par le pouvoir politique ou par le grand public, y compris jusque dans leurs implications économiques. Qu'est-ce que la politique sinon un certain nombre d'initiatives qui vont influencer l'évolution économique? L'approche de cette recherche sur le droit du Travail est certes intéressante mais il faudrait certainement l'enrichir considérablement sur le plan de l'analyse de l'environnement et de l'analyse du jeu d'acteurs parce qu'il y a dans l'élaboration de ces textes un jeu d'acteurs qui est un jeu de rapports de force. N'oublions pas, lorsqu'on parle de juridisme, que le droit du Travail est un droit pénal. Le droit pénal est dans notre pays d'interprétation stricte, et comprend donc un aspect extrêmement pointilleux juridiquement, ce qui rend d'autant plus utile d'élargir l'analyse à tous ces jeux d'acteurs.

Pour illustrer, on pourrait prendre l'exemple des directives européennes qui touchent à la santé au Travail. Certaines de ces directives sont qualifiées de «sociales», d'autres se rattachent à la construction du marché unique et sont considérées comme économiques. J'évoquerai l'exemple que je connais le mieux: celui des directives concernant, respectivement, d'une part l'utilisation des machines et autres équipements de travail, ou des équipements de protection individuelle, et d'autre part la conception et la mise sur le marché de ces mêmes équipements. Dans les deux cas, les négociateurs de la direction des relations du Travail ont eu le souci de 
tenir les deux bouts de la chaîne: assurer le niveau de sécurité le plus élevé possible, défendre notre industrie nationale. Dans les deux cas, ils ont travaillé en étroite concertation avec les partenaires sociaux. Dans les deux cas, force est de reconnaître que le Parlement n'a exercé qu'une influence limitée, ce qui est certainement regrettable du point de vue démocratique, et du point de vue de la perception des acquis positifs de l'Union européenne, par rapport à d'autres «acquis communautaires» plus discutables, et peut-être moins concertés.

\section{- Jean-Pierre Le Crom}

Avant 1914, la direction du Travail est le pivot d'une action au sein de ce que Christian Topalov appelle une "nébuleuse réformatrice» composée de parlementaires, d'associations, d'institutions comme le Musée social, comme l'Association pour la protection légale des travailleurs, etc. , mais dans lesquels les syndicats jouent un rôle faible. Certains syndicalistes jouent un rôle parce qu'ils participent à l'Office du travail, au Musée social, etc. : en résumé, il s'agit surtout de relations interpersonnelles. Après la Première Guerre mondiale, c'est plutôt ce que Pierre Laroque appelait une "réglementation à base contractuelle» qui se met en place, en matière de durée du travail mais pas seulement. On demande aux acteurs sociaux de se mettre d'accord et l'État accompagne: les syndicats et les organisations professionnelles, avec le Conseil national économique et les lois de 1936, jouent un rôle tout à fait important. La nébuleuse réformatrice n'a peut-être pas entièrement disparu, mais un nouveau modèle se substitue au premier. Pour reprendre la distinction entre technique et politique évoquée par Daniel Lejeune, il existait une différence entre les conseils, certains étant plus politiques que d'autres. Il prenait l'exemple de la Commission supérieure des conventions collectives, considérée comme une caisse de résonance des mots d'ordre syndicaux et en fait politiques. En revanche, il existe des conseils qu'on connait moins, plus techniques en matière d'hygiène et sécurité, et le rôle joué par ces conseils est fondamental.

\section{- Claude Chetcuti}

Il est important d'examiner quels sont les outils juridiques utilisés par la puissance publique pour faire appliquer la législation car ils peuvent être divers. En ce qui concerne la santé au travail, on prétend en 1945 pouvoir instaurer un système de prévention des accidents du travail réglant de façon rigide la mise sur le marché et l'utilisation de toute machine dangereuse. C'est le système de l'homologation inscrit dans le Code du travail, assorti de sanctions pénales en cas d'infraction. Mais le ministère n'a pas les moyens de sa politique et très vite le système s'enraye. Le relais est pris par le Code de la sécurité sociale: les caisses régionales d'assurance-maladie et la caisse nationale pourront édicter des mesures générales de prévention sanctionnées financièrement. Avec la loi du 6 décembre 1976, on revient à la pénalisation et les employeurs refusent désormais leur participation à l'élaboration des mesures générales, désormais assorties d'une sanction pénale. Ainsi, suivant les outils utilisés dans le Code du travail ou celui de la Sécurité sociale, les leviers d'intervention au niveau des entreprises seront distincts, mis en œuvre par des agents différents, perçus différemment et auront une efficacité différentiée.

\section{- Témoignage de Pierre Grandjeat, président de chambre honoraire à la Cour des comptes}

J'interviendrai essentiellement à propos de la recherche sur le personnel du ministère du Travail, à deux titres. J'ai été au cabinet de Gilbert Grandval, il y a maintenant plus de quarante ans. Et je suis aussi énarque des premières promotions. Je crois que personne n'a évoqué l'intervention éventuelle de l'ENA dans la structure du ministère du Travail. Lorsque j'étais à l'ENA il y a cinquante-quatre ans, la «section sociale» avait uniquement comme débouché naturel le ministère du Travail, le ministère de la Santé et celui des Anciens combattants. Pour nous présenter le ministère du Travail, nous avons eu en face de nous $\mathrm{M}^{\text {elles }}$ Raffalovich et Petit. Quand la présentation a été terminée, aucun d'entre nous - nous étions onze - n'avait envie d'aller au ministère du Travail! Non pas parce que ces personnes n'étaient pas de qualité, mais parce que le tableau qu'elles nous faisaient de leur activité relevait du juridisme pur, ce qui ne tentait pas les gens de ma génération. Le ministère du Travail était tenu par des personnes qui étaient là depuis longtemps sans l'intention d'en partir, et où les nouveaux énarques avaient peu de chance de trouver un accueil favorable et de faire carrière. Je parle au nom de ma promotion, mais les promotions d'avant et d'après, me semble-t-il, ne différaient guère sur ce plan-là.

Le second point est lié à cette question du «juridisme» du ministère et au témoignage d'Annie Fouquet, qui a évoqué la création du CEREQ en 1971 et le rôle de l'inspection du Travail. Je crois que le premier nouveau concours de l'inspection du Travail a été créé en 1955. Il n'y en avait pas eu depuis 1946. La création par Pierre Laurent, directeur du Travail, du nouveau concours des inspecteurs du Travail a été un moyen de faire évoluer les mentalités. Mais il a quitté le ministère du Travail pour devenir secrétaire général du ministère de l'Éducation nationale. C'est à partir de cette double expérience qu'ont germé l'idée et la création du CEREQ, et qu'est apparue l'importance de la prévision dans un domaine qui jusqu'alors avait été assez juridique. Pierre Laurent connaissait bien le sujet et a souhaité aller au-delà de l'expérience qu'il avait acquise au ministère de l'Éducation. Il a demandé à Gabriel Ducray, major de la première promotion des nouveaux inspecteurs du Travail, de le rejoindre 
au ministère de l'Éducation nationale où ils ont créé ensemble le nouveau Service des études et statistiques (SES) dont G. Ducray a été le premier responsable avant de devenir, quelques années plus tard, le créateur et responsable du CEREQ. Ainsi, grâce à $\mathrm{P}$. Laurent et G. Ducray a été mis en place pour la première fois un lieu et un outil permettant de corréler la formation initiale et les besoins de l'économie en emplois.

Table ronde no 2
Acteurs et réseaux
du ministère du Travail
Présidente : Catherine Omnès
Recherches présentées
Anne Revillard, CNRS/ENS Cachan, La cause
des femmes au ministère du Travail: le Comité du
travail féminin (1965-1984).
Lucie Tanguy, CNRS, Paola Cristofalo,
Laboratoire travail et mobilité, L'institut des
sciences sociales du travail. Contribution à l'his-
toire de la sociologie du travail en France (1950-
1970).
Jean-Claude Barbier, CNRS-MATISSE
université de Paris-I-Panthéon-Sorbonne, Alfred
Golliard, préfet résistant (1881-1944). Matériaux
pour une biographie.

\section{Discussion par Marie-Thérèse Letablier, Centre d'études de l'emploi}

Les trois rapports portent sur les acteurs et les réseaux du ministère du Travail. La cohérence entre eux vient de leur objet. J'ai été assez enthousiasmée par le travail sur le Comité du travail féminin, d'autant que l'auteure a su capter l'atmosphère qui imprégnait ce comité, auquel j'ai eu l'occasion de participer alors que j'étais très jeune chercheuse au Centre d'études de l'émploi. Le Comité du travail féminin a permis d'accompagner cette révolution silencieuse du travail des femmes à une période clé de cette évolution. Il a su proposer des réformes grâce à sa capacité d'expertise et à sa liberté de paroles articulée à un fort consensus entre les forces sociales investies dans ce comité. Je m'interroge sur la manière dont le comité a pu gérer l'attention entre une sorte de logique militante et une logique administrative. Est-ce que la liberté de paroles du CTF n'a pas été la raison de sa disparition, même si bien d'autres raisons expliquent cette dernière? En quoi la notion de «féminisme d'État» est-elle utile?
A-t-on des éléments de comparaison avec ce qui se passe dans d'autres pays comme le Québec?

La recherche sur l'ISST nous apprend beaucoup de choses, et notamment le chapitre consacré à l'action du ministère du Travail en direction des syndicats, qui décrit très minutieusement la mission sociale aux États-Unis sur les cinquante-cinq qui ont lieu entre 1950 et 1957 sous l'égide du ministère $\mathrm{du}$ Travail. Une première interrogation concerne les raisons pour lesquelles l'ISST a cessé de fonctionner, alors que périodiquement renaît l'idée d'un tel organisme dans le débat général. Je me suis aussi demandé pourquoi ce courant des relations professionnelles des années 1950-1960 n'a pas totalement réussi à s'imposer dans le champ académique comme aux États-Unis ou en Grande-Bretagne, où il constitue une discipline plus autonome qu'elle ne l'est en France. Il serait intéressant de revenir sur la question des relations entre l'ISST et le CNRS.

Le troisième rapport diffère des deux précédents puisqu'il s'agit d'une biographie qui traite des rapports entre les instances administratives locales et le ministère du Travail. J'aurai une question relative à la gestion des réfugiés allemands à Strasbourg en 1933-1934. La gestion du contrôle, de l'accueil et de l'administration des réfugiés Allemands, Juifs, pour la plupart, qui quittent l'Allemagne et arrivent en Alsace au début de 1933, incombe à la préfecture, dont l'action est analysée avec beaucoup de précisions. Face à l'obligation d'accueillir les réfugiés, le préfet du Bas-Rhin et son secrétaire général se construisent une sorte de doctrine ou ligne de conduite à partir d'idées et de représentations qui sont celles de leur époque et de leur milieu social. Mais cette doctrine relève aussi de leurs orientations personnelles et de leurs valeurs. La comparaison entre trois préfectures d'Alsace permet d'étayer cette hypothèse, en soulignant la diversité des modes de gestion de cette question dans les trois préfectures, en lien avec la diversité des doctrines dans les trois départements, alors qu'ils sont soumis aux mêmes contraintes et en priorité au rôle des chambres de commerce locales sur la question de la main-d'œuvre étrangère. Le gouvernement central n'a pas de doctrine sur ce sujet et laisse les préfets sans instructions. L'approche biographique pour traiter de ces questions comporte des difficultés particulières: ne comporte-t-elle pas le risque de laisser dans l'ombre, en se focalisant sur un acteur singulier, des acteurs collectifs, et les «nébuleuses réformatrices» qui peuvent se trouver autour de ces institutions locales?

\section{- Anne Revillard}

La tension entre logique militante et logique administrative est structurante. Elle se nourrit de la présence de syndicats et d'associations d'acteurs militants dans son sein, avec d'ailleurs, des conflits internes fréquents, puisque les syndicats, les associations familiales et féministes de l'époque ne sont 
pas forcément d'accord sur les questions relatives au travail des femmes. Mais elle se nourrit aussi du militantisme qui a présidé à la création du CTF. Ses fondateurs se sont en effet inspirés de l'exemple américain du Womens'Bureau; mais les associations féminines de droite, créées dans l'après-guerre, qui avaient un ancrage dans le milieu associatif féminin ont aussi joué un rôle fondamental. Le Comité a été investi par des personnes qui avaient des objectifs précis en matière de réformes du droit du travail pour ce qui concerne le statut des femmes. C'est pourquoi il a très rapidement adopté une démarche revendicative vis-à-vis du ministère du Travail.

Comment est-ce que le ministère gère cette tension? Ce qui gêne, ce n'est pas tant la radicalité des positions défendues que la manière dont elles sont portées auprès du ministère du Travail; c'est le fait qu'on s'adresse au ministère du Travail sur un ton revendicatif, qui n'est pas conforme au langage administratif souhaité. La tension se résout dans la préservation de la radicalité du contenu de certaines demandes, mais sous une forme adaptée à la formulation administrative. Ce qui compte, c'est de parler un langage administratif et notamment de parler un langage d'expert; c'est essentiel, non seulement parce qu'ily a un enjeu dans la production de savoirs sur le travail des femmes, mais aussi du point de vue de la posture adoptée vis-à-vis du ministère. Le CTF a toujours insisté sur cette logique, purement administrative et experte, alors que sur le fond, il porte des demandes de réformes qui peuvent être d'une assez grande ampleur.

La liberté de parole du CTF n'est pas la raison de sa disparition. Il a été supprimé par la ministre Yvette Roudy qui a pour particularité d'être elle-même une ancienne féministe. C'est en revanche l'opposition gauche-droite qui a joué. Le CTF était perçu comme de droite par son origine, même s'il avait aussi des acteurs de gauche en son sein, et même si ses prises de positions sur les questions de travail des femmes n'étaient pas conformes au familialisme de droite. Le Conseil supérieur de l'égalité professionnelle qui lui est postérieur n'a pas eu du tout le même statut, bien qu'il ait aussi, potentiellement un peu un rôle de prospective: il n'était pas dans la même posture critique vis-à-vis des politiques existantes. Au Québec, en revanche, le Conseil du statut de la femme épouse la même posture d'analyse critique et de promotion de réformes. En France, les structures qui sont apparues après la disparition du CTF ont été de type ministériel plus classique, et ont assumé un statut administratif plus marqué. Par ailleurs, le terme de «féminisme d'État» est certes connoté, mais il désigne un champ de recherche en sciences politiques, qui s'est développé notamment sous l'impulsion de chercheuses nord américaines depuis les années 1990 travaillant sur les instances gouvernementales.

\section{- Lucie Tanguy}

Pour comprendre la question de la disparition de l'ISST, il faut revenir sur les conditions d'existence de cette institution créée en 1951 comme une institution d'enseignement destinée aux spécialistes des questions du travail dans les entreprises. Il est essentiel de rappeler qu'il s'agit d'une direction collégiale, et que le ministère du Travail discute âprement sa place dans la prise de décision. Il faut rappeler aussi qu'il y a un financement exceptionnel qui vient de l'aide américaine, sans rapport avec ce qui existe au CNRS à l'époque. Les conditions de travail ne sont pas celles des chercheurs du CNRS en sciences sociales, qui à l'époque font leurs travaux chez eux, avec un local pour se rencontrer. L'ISST a des bureaux et des moyens techniques, on y bénéficie de l'appareil statistique du ministère du Travail. Il faut rappeler aussi que c'est une division du travail très hiérarchisée: au sommet, il y a des chercheurs très titrés qui sont attachés au CNRS mais qui font leurs recherches dans l'ISST. C'est le cas de Crozier, de Reynaud, de Touraine, etc. Ces chercheurs sont responsables des recherches et sont les interlocuteurs directs des politiques et des administrateurs; ce sont de véritables entrepreneurs de recherches. L'institution n'a pas disparu si vite mais plusieurs éléments d'explication sur sa disparition coexistent: le tarissement de cette manne que représentait l'aide américaine; le fait que se soit constitué un groupe de chercheurs qui y ont appris leur métier et qui aspirent à une plus grande indépendance. L'héritage de l'ISST n'a pas pour autant disparu, parce que tous ces chercheurs vont fonder après d'autres équipes de recherches. Le premier héritage, c'est une sociologie empirique: c'était le projet des chercheurs de l'après-guerre. Mais l'ISST va être un lieu plus favorable pour fonder une sociologie empirique sur la formule survey research avec de vastes enquêtes et une validation par les instruments statistiques, une sociologie qui est en prise sur l'action avec des visées prédictives. Pour autant, contrairement à ce qui a été donné par le ministère du Travail comme missions à cette institution, il ne s'agit pas d'une recherche appliquée qui donnerait des conseils aux entreprises. La revue Sociologie du Travail sort de cette institution. Elle devait même être publiée par cette institution. On retrouve les chercheurs de l'ISST dans les comités de rédaction et tous les premiers numéros, les notes critiques, les comptes rendus sont fait par les chercheurs de cette institution.

\section{- Jean Claude Barbier}

Nous avons tenté avec Olivier Bütner de nous mettre, en tant que sociologues, aux méthodes historiques. En ce qui me concerne, je me suis intéressé à un aspect que les sociologues abordent rarement: la place réelle de l'individu dans un système. La marge de manœuvre est un classique dans la sociologie des organisations, dans la sociologie des politiques 
publiques, mais la marge individuelle est beaucoup plus difficile à étudier. L'avantage de la démarche biographique, c'est le fait que l'on peut avoir accès à des sources privées. Une thèse de Vicky Caron, Amnésie et Silence, explique l'importance du rôle des classes moyennes dans la façon dont le gouvernement a traité la question des réfugiés. Nous arrivons aux mêmes conclusions, mais les matériaux que j'ai explorés ne permettent pas de confirmer la thèse selon laquelle les petits fonctionnaires interprèteraient les choses à leur façon. On s'aperçoit que sur place, au plan local, il n'existe pas de politique, donc, forcément, il n'y a rien à appliquer. Il est intéressant de voir comment une doctrine se construit, à partir des examens des études de cas : sur le plan méthodologique, il s'agit donc de repérer dans les écrits du secrétaire général les initiatives qu'on peut lui attribuer, initiatives d'ailleurs qu'il prend souvent, sous couvert du préfet.

Une dernière chose nous a frappés, c'est le caractère politique de la mise en œuvre des politiques. Par exemple, sur la mise en œuvre des 40 heures, alors que le Jura a voté en 1936 en majorité pour les opposants du Front populaire, il y a une sorte de coup d'état local du Parti radical pour évincer un candidat qui aurait été plus dans la ligne du Front populaire; le préfet se trouve ainsi placé devant la nécessité d'engager la politique du gouvernement. Il devient la bête noire des notables locaux, et notamment du sénateur Dumont qui s'oppose aux 40 heures.

\section{- Catherine Omnès, université Versailles- Saint Quentin en Yvelynes}

Claire Zalc a fait son DEA sur les réfugiés allemands à partir des archives de la chambre de commerce qui confirme le «déséquilibre» qui s'est établi entre la chambre de commerce et la préfecture à ce sujet.

\section{- Témoignage de Jacques Freyssinet}

Mon objectif est de repérer d'éventuelles intersections entre les travaux qui ont été présentés au cours de cette table ronde et les expériences que j'ai vécues. Une question qui leur est commune porte sur les relations entre le ministère du Travail, le monde de la recherche et le débat social. Elle est directement présente dans les deux premiers rapports, portant respectivement sur l'Institut des sciences sociales du travail (ISST) et sur le Comité du travail féminin (CTF). En revanche, pour le troisième rapport concernant le préfet Golliard, il est impossible d'appliquer cette problématique, quel que soit par ailleurs l'intérêt passionné que l'on peut prendre à l'analyse des grèves dans l'entre-deux guerres à Strasbourg. Je voudrais, dans un premier temps dégager les enseignements partiels que j'ai tirés des deux premiers rapports pour les appliquer à d'autres expériences que j'ai connues et réfléchir sur leur cohérence ou discordance. En préambule, je voudrais rappeler qu'avant le ministère du Travail, il y avait, dès 1891, l'Office du travail qui constituait une structure où l'accumulation des connaissances était réalisée sous le contrôle d'un débat tripartite. Le besoin d'articuler l'accumulation des connaissances et le débat avec les acteurs sociaux n'est donc pas une découverte récente pour le ministère; il est à l'origine de sa création.

D'abord, quelques enseignements tirés des rapports. Sur l'ISST, l'histoire de sa «section recherche» a été pour moi une découverte. Il s'agissait, au sein de l'université, d'une émanation directe du ministère du Travail qui a eu l'initiative de sa création, grâce à certains de ses hauts fonctionnaires. Le contexte pouvait faire craindre le pire, comme nous l'explique Lucie Tanguy, quant à l'indépendance, donc à la qualité de la production des connaissances. D'abord, l'initiative se situait dans le prolongement des «missions de productivité» qu'organisait alors le ministère aux ÉtatsUnis, avec un objectif affiché de promotion des «syndicats libres» et des relations sociales pacifiques au service de l'efficacité productive. Ensuite, le financement provenait essentiellement de fonds américains, ce qui, dans le contexte de la guerre froide, créait un effet de signal sans ambiguïté. Et pourtant... L'expérience ne permet pas de mettre en doute l'indépendance de l'équipe des chercheurs qui, sous l'égide de Georges Friedmann, a réuni des gens comme Alain Touraine, Jean-Daniel Reynaud, Claude Durand et d'autres. Alors que la création de la «section recherche» pouvait sembler répondre à une demande utilitaire de court terme émanant du ministère, celle-ci a produit des travaux qui ont amené en France des avancées méthodologiques importantes: premières recherches lourdes de terrain avec entretiens approfondis, recueil et exploitation de statistiques originales, restitution et discussion des résultats avec les acteurs concernés... Enfin, c'est le même groupe qui, à partir de la «section recherche», est à l'origine de la création de la revue Sociologie du Travail dont on sait l'impact durable qu'elle a exercé sur le développement de la discipline. De manière paradoxale, on passe donc des missions de productivité et des fonds américains à un investissement lourd du ministère du Travail dans un organisme de recherche qui a des effets structurants sur les questionnements et la méthodologie des analyses concernant le travail.

La deuxième expérience est celle du Comité du travail féminin, retracée par Anne Revillard. Elle est bien différente de la première. D'abord, il ne s'agit plus ici d'une émanation de l'appareil du ministère, ni d'une initiative de hauts fonctionnaires. $\mathrm{Au}$ contraire, la création du Comité est quasiment imposée de l'extérieur, sous l'influence de mouvements associatifs articulés à des mouvements politiques, qui, à un moment donné, obtiennent l'appui du ministre. Ensuite, l'institution a toujours du mal à trouver sa place au sein du ministère, à y imposer 
sa légitimité et à y obtenir des moyens. Pourtant, elle défend jusqu'au bout son appartenance au ministère, contre les tentatives de réaffectations externes. Cet attachement s'explique par le fait que les animatrices du CTF, même si leur origine les associait plutôt au centre-droit, avaient la conviction que c'était à partir de l'accès au travail rémunéré que pouvaient progresser le statut et la position sociale des femmes. De fait, lorsque le CTF sortira du ministère, ça sera pour en mourir. Enfin, c'est une institution qui, dès le départ, tire sa légitimité d'une part, de la production de connaissances, en tout cas, de sa capacité à mobiliser le monde de la recherche et, d'autre part, de l'expérience de ses membres dans le champ social, de leurs responsabilités reconnues en tant qu'acteurs sociaux. Le Comité utilise ces connaissances à des fins d'intervention politique, de conseil, d'influence, de lobbying, mais aussi, de façon plus ambitieuse, pour faire évoluer les représentations des acteurs. Il est traversé de débats contradictoires, parfois vigoureux, qui se poursuivront de longues années sans provoquer son explosion, ni sa mise en sommeil. Le ministère du Travail, en tant qu'administration, n'a pas été à l'origine du Comité, dont l'activité ne l'a visiblement jamais enthousiasmé, mais il a tout de même été un lieu d'accueil pour une confrontation durable entre des acteurs qui considéraient la recherche comme un outil indispensable pour construire leurs diagnostics, leurs revendications et leurs stratégies.

Ensuite, quelques leçons de l'expérience. En lien avec ces remarques, j'évoquerai quatre expériences que, par les hasards de l'existence, j'ai été amené à vivre. De 1981 à 1987, j'ai été président du conseil d'administration de l'ANPE. Parmi ses responsables, j'ai retrouvé notamment Gérard Vanderpotte, son directeur général, Bernard Mériaux qui était son numéro deux, et Vincent Merle qui était directeur des études et de la statistique. Or, pendant des années, nous avions travaillé ensemble au sein du séminaire «Emploi» du commissariat général du Plan, lieu qui avait joué, sous l'impulsion de Bernard Mériaux, un rôle important de confrontation entre monde de la recherche et responsables de l'administration. Nous étions marqués par cette expérience au cours de laquelle les résultats de la recherche avaient servi d'aliments aux réflexions sur la politique de l'emploi. Fidèle à cette logique, l'ANPE lance deux appels successifs qui financeront un ensemble de recherches sur le fonctionnement des marchés du travail et l'action des intermédiaires du marché du travail. Une «itération» assez stimulante s'est ainsi opérée entre orientations de recherche et politiques de l'emploi. Quels enseignements en tirer? Un simple moment privilégié? Une configuration astrale assez invraisemblable qui ne pouvait pas durer et qui n'a d'ailleurs pas beaucoup duré? Oui, mais en même temps, je n'ai jamais perçu de réticences face à ces démarches de la part de notre administration de tutelle qui était la
Délégation à l'emploi. Les délégués à l'emploi que j'ai pu connaître, de Gabriel Mignot à Dominique Balmary, ont bien accepté cette démarche, ce qui me semble illustrer la tradition d'ouverture à la recherche toujours vivante dans ce ministère.

La deuxième expérience concerne le Conseil scientifique de l'évaluation des politiques publiques. En 1990, un dispositif interministériel de l'évaluation est créé avec, d'une part, un Comité interministériel, qui passe commande et met en œuvre, le cas échéant, les résultats et, d'autre part, un Conseil scientifique, qui est chargé de vérifier l'indépendance, la transparence et la rigueur scientifique des opérations. J'ai fait partie de cet organisme pendant les six premières années de son existence. Dans ce cadre, je suivais principalement les dossiers relatifs à l'emploi et à la formation professionnelle et je retiendrai l'exemple de l'évaluation de la loi quinquennale sur le travail, l'emploi et la formation professionnelle de décembre 1993, car il montre bien le souci des hauts fonctionnaires du ministère de mobiliser les ressources de la recherche. Claude Seibel, directeur de la DARES, fait introduire dans la loi un article qui prévoit une évaluation interministérielle, ce qui signifie que le ministère du Travail renonce à la commodité de l'auto-évaluation et se soumet à une procédure d'évaluation publique. Le président de l'instance d'évaluation est Pierre Cabanes, ancien délégué à l'emploi, et le rapporteur général Françoise Bouygard, aujourd'hui déléguée adjointe à l'emploi et à la formation professionnelle. Le Comité lance un appel d'offres; onze études sont commandées à des équipes universitaires ou à des cabinets de conseil. C'est, à ma connaissance, le premier ensemble, indépendant et rigoureux, d'évaluation d'un ensemble de dispositifs de la politique de l'emploi et c'est à l'initiative du ministère du Travail qu'on le doit. Ce dispositif interministériel a d'abord été réformé, puis supprimé, ce qui était extrêmement regrettable.

Une troisième expérience a été lancée au sein de l'Institut national du travail (INT) au début des années 1980. Jean Cordouan, directeur de l'INT, crée des sessions tripartites qui s'étalent sur plusieurs mois et réunissent des participants provenant des organisations syndicales, des entreprises et de l'administration. Ils sont appelés à confronter leurs points de vues à partir d'une gamme d'expériences, nationales et étrangères, éclairées par les apports de différents courants de recherches. J'ai participé pendant un certain nombre d'années au comité de pilotage et je dois dire que son indépendance a toujours été respectée. Dès le départ, il était entendu que l'ensemble des participants étaient producteurs de connaissances. Ils n'étaient pas là simplement pour absorber un enseignement, mais pour produire une analyse qui se traduisait par un rapport final de très bonne qualité. Le ministère du Travail a soutenu cette démarche, acceptant le risque d'une confronta- 
tion pluraliste et contradictoire entre acteurs sociaux et chercheurs. Cela marche encore aujourd'hui.

Enfin, la quatrième expérience que je souhaite évoquer est celle de l'Institut de recherches économiques et sociales (IRES), institut chargé de répondre aux demandes exprimées par les organisations syndicales en matière de recherche. L'IRES se trouvera en relation avec le ministère du Travail de différentes façons. Tout d'abord, ses représentants au conseil de l'IRES (Claude Seibel, puis Annie Fouquet, pour la période que j'ai connue) y ont joué un rôle particulièrement actif et positif. Ensuite, l'IRES a réalisé de nombreuses études ou recherches contractuelles pour le ministère dans le plein respect de sa mission spécifique et de son autonomie. Deux enseignements plus généraux peuvent en être tirés. En premier lieu, l'IRES, comme toutes les équipes de recherche, s'est trouvée confrontée au rôle de plus en plus fort que jouent les appels d'offres comme mode de pilotage de la recherche par les ministères, ce qui pose des questions importantes, avec des aspects positifs et négatifs, sur lesquelles il n'est pas possible d'avoir de diagnostic simpliste et qu'il serait trop long de développer ici. Ces questions relèvent bien des interrogations que nous avons aujourd'hui sur les rapports entre ministère du Travail et monde de la recherche. En second lieu, cette expérience pose aussi la question du positionnement de la DARES, puisque cette dernière a été créée, sur le rapport de Gabriel Mignot, comme direction d'animation de la recherche. Cette orientation de principe n'a pas été remise en cause, mais on voit bien que la dynamique d'une institution de ce genre pose deux types de problèmes. D'abord, il est difficile, à la longue, à des responsables chargés d'animer la recherche de ne pas être tentés d'avoir une conception relativement active, voire stimulante, à la limite contraignante, de cette «animation». Il y a là une source de tension possible avec le monde de la recherche. Ensuite, il existe le risque que la DARES, sur la base de cette responsabilité, devienne un sas ou un écran entre le monde de la recherche et l'ensemble des directions du ministère, de telle sorte que finalement, l'influence de la recherche sur la réflexion du ministère du Travail puisse être moins directe qu'on ne pouvait l'espérer.

En conclusion, les rapports présentés comme l'expérience vécue confirment l'hypothèse qu'il existe une tradition forte, quoique discontinue, du ministère du Travail. Il a su se donner les moyens d'un double dialogue, partiellement interdépendant, avec d'une part, les acteurs sociaux, d'autre part, le monde de la recherche. La démarche implique l'acceptation des principes du pluralisme et du contradictoire. Ces tentatives apparaissent sous des formes multiples et connaissent des succès inégaux, avec des émergences improbables et des destins imprévisibles. Il est difficile d'en construire un schéma explicatif global, mais leur analyse est riche d'en- seignements, autant par leurs difficultés, voire leurs échecs, que par leurs réussites, même provisoires.

\section{- Jean-Pierre le Crom}

Il y a un domaine où le rôle des préfets, des souspréfets et des secrétaires généraux de préfecture en matière de droit du Travail est très important, c'est celui de la conciliation et de l'arbitrage des conflits du travail, avec une grande différence entre avant 1936 et après. Avant 1936, on est sous l'empire de la loi de 1892 sur la conciliation et l'arbitrage des conflits du travail, loi libérale qui donne un rôle très important aux juges de paix. Mais les juges de paix ont une compétence limitée aux cantons. Pour cette raison, on voit apparaître à partir de la fin du XIX ${ }^{\mathrm{e}}$ jusque dans les années 1930 une deuxième forme de conciliation, plus informelle où les préfectures aux différents niveaux jouent un rôle tout à fait important. Concernant le CTF, on a vraiment le sentiment, à partir des nombreux travaux qui ont déjà été écrits sur le rôle des conseils, des comités, des commissions dans l'administration, et sur la consultation dans l'administration notamment celle du Travail (Yves Delamotte a fait un article sur le sujet) que ce comité tranche et qu'il est assez exceptionnel par rapport aux autres.

\section{- Bernard Laurençon, président du Comité d'histoire de Lyon}

J'interviendrai au titre de secrétaire général de l'Association des auditeurs de l'INT, qui fédère les auditeurs ayant participé aux sessions nationales, organisées depuis 1986 et qui, effectivement, permet à des cadres d'entreprises, syndicaux ou des administrations de dialoguer sans emprise hiérarchique. Je peux témoigner que nous avons créé cette association en 1987 pour poursuivre ce travail car les sessions sont terminées. Il y a actuellement cinq cent trente personnes adhérentes qui ont eu l'occasion de participer à ces sessions, deux cents qui continuent de cotiser régulièrement à l'association et nous nous retrouvons toujours pour faire des voyages d'études et organiser des visites d'entreprises sur le thème du dialogue social. Grâce à cette continuité qui a été initiée par le ministère du Travail et par l'INT avec Jean Cordouan, un certain nombre de réseaux se sont établis, un certain nombre de conflits se sont résolus grâce aux connaissances et aux liens établis à l'occasion de ces sessions.

\section{- Pierre Maclouf, CERSO Paris-Dauphine}

Il me semble que le comité du travail féminin est original parce qu'il prend en charge aussi un secteur de problèmes qui a une grande importance. Mais il ne faut pas oublier que par nature, le ministère est en relation avec différentes instances, avec des organismes qui ont une logique militante et revendicative, avec les syndicats dans la Commission supérieure des conventions collectives, dans les instances qui entretiennent avec lui une relation qui 
est à la charnière des revendications militantes et $\mathrm{du}$ souci de trouver une traduction juridique à ces revendications. De la même façon, les organismes avec lesquels le ministère est en relation se dotent aussi de centres de recherches propres (des centres d'études économiques confédérales ou un bureau de recherches d'action économique pour la CFDT, par exemple). Et il faudrait voir comment ces instances de recherches, propres aux centrales syndicales, entrent en relation avec la production de la sociologie du travail.

\section{- Pierre Grandjeat}

Est-ce que le Comité du travail féminin a eu à débattre ou à connaître des problèmes importants qui ont concerné les femmes dans cette période 1965-1981, à savoir les problèmes de la contraception et d'avortement avec les lois Neuwirth et Veil, qui étaient liées et pouvaient avoir un impact important sur le déroulement du travail et la carrière des femmes?

\section{- Anne Revillard}

Le Comité est resté étrangement silencieux sur les questions de contraception et l'avortement. J'ai deux hypothèses sur ce silence. La première est liée à l'existence de dissensions internes au Comité sur cette question. La deuxième, c'est que le Comité choisissait ses batailles et qu'il voulait conserver sa légitimité vis-à-vis du ministère du Travail. Par ailleurs, le ministère du Travail a toujours été en relation avec des organismes revendicatifs, mais ce qui est nouveau, c'est que des personnes salariées du ministère du Travail soient dans ce type de posture, le comité ayant des permanentes issues de l'administration du ministère du Travail.

\section{- Catherine Omnès}

Concernant le fond d'archives Jean Gouin, sait-on comment s'articulent les missions de productivité aux États-Unis et l'intérêt pour d'autres modèles que le modèle américain? S'intéresse-il à l'URSS, aux pays nordiques et à Allemagne?

\section{- Lucie Tanguy}

En fait, ils vont voir le modèle américain, parce que les fonds sont destinés à cela. D'après les courriers, s'ils avaient eu des fonds pour observer d'autres pays, ils l'auraient fait. En particulier, j'ai étudié une mission faite en 1953 en Norvège, Suède, Finlande, où les conclusions sont presque les mêmes. Nous n'avons cependant pas fait une exploitation systématique de ce fonds.

\section{- Isabelle da Costa, Centre d'études de l'emploi}

Lorsqu'on sait ce qu'était dans les années 1950 le mouvement ouvrier américain (les grèves liées au contrôle de la production), si on avait dit, à l'époque, à un leader syndical américain qu'il était coopératif avec le patronat, il aurait pris ça pour une injure et le modèle adversarial était dominant dans le mouvement ouvrier américain, d'où ma question: comment étaient organisées ces missions?

Quelles personnes étaient rencontrées pour que de telles idées ou interprétations soient rapportées?

\section{- Paola Cristofalo}

C'est en fait beaucoup plus nuancé et cela dépend des missions. Pour certaines missions, ils disent vraiment que les syndicats ne coopèrent pas mais qu'ils tirent partie de l'augmentation de la productivité si elle se traduit en termes de salaire. Il y a beaucoup d'éléments de propagande dans les rapports des missions. On voit parfois que certaines missions ne se passent pas très bien parce que les syndicalistes français ne sont pas acceptés dans les entreprises qu'ils sont censés aller voir. Certains employeurs laissent seulement faire les entretiens, mais pas les visites dans les services techniques des syndicats. C'est donc très hétérogène. 


\section{Table ronde $n^{\circ} 3$ \\ Le ministère du Travail et les organisations professionnelles et syndicales}

Président: Udo Rehfeldt (IRES)

\section{Recherches présentées}

Michèle Tallard, CNRS-IRIS-CREDEP, Catherine Vincent, IRES, Olivier Giraud, CNRSCURAPP-Centre Marc Bloch, Michèle Dupré, GLISY-SAFA, Les relations État-acteurs sociaux en France et en Allemagne.

Jean Saglio, université Pierre-MendèsFrance, Grenoble-Laboratoire CRISTO, Sophie Le Corre, CNRS-CNAM, Le ministère du Travail et les secteurs tertiaires. La régulation des relations professionnelles dans le secteur du grand commerce alimentaire de 1950 à nos jours.

\section{Discussion par Éric Pezet, Centre de gestion de l'École des mines, université d'Évry Val-d'Essonne}

Les deux rapports peuvent être rapprochés du point de vue des niveaux auxquels ils étudient la relation entre l'État et les syndicats salariés et syndicats d'employeurs. Le premier rapport me paraît porter sur le débat public et la décision politique, tandis que le second porte davantage sur la capacité à agir sur le terrain.

Sur la période étudiée par Michèle Tallard, Catherine Vincent, Olivier Giraud et Michèle Dupré, dans les deux pays, la France et l'Allemagne, la question centrale est celle de l'articulation entre la politique économique et la régulation que peuvent produire les syndicats et le patronat par la négociation. C'est la question du contenu de la démocratie industrielle. Il existe différentes formes possibles: le contrôle de l'entreprise, la participation, la cogestion, l'autogestion. En France, le concept de démocratie industrielle n'a pas le même sens pour toutes les organisations syndicales. Pour la CFDT, il s'agit bien de démocratiser l'entreprise. Qu'est-ce que la démocratie industrielle pour les différentes organisations syndicales? Par ailleurs, selon le rapport, la position du patronat sur cette question de la démocratie industrielle est finalement assez simple: le patronat n'en veut pas. Il y a pourtant, au sein du patronat, des différences d'approches de la démocratie industrielle. Certaines branches comme celle du commerce de gros participent peu aux débats, elles ont du mal à se constituer, mais d'autres, comme la métallurgie, sont au contraire très actives. Il y a aussi des réformes politiques qui sont très en lien avec les pratiques d'entreprises. On peut citer l'exemple de la mensualisation qui est une réforme mise en œuvre sous la présidence de Georges Pompidou. Celle-ci a été réalisée alors que de nombreuses entreprises pratiquaient déjà ce mode de paiement des salaires. Si on prend les cas de Renault, de Berliet: ces entreprises ont un rapport très fort à l'institutionnalisation de la démocratie industrielle mais ce rapport est différent. Renault est un laboratoire social qui inspire certains accords; Berliet est une entreprise où l'affrontement entre le patronat et les syndicats est violent mais où la négociation est néanmoins importante. Et surtout, c'est l'entreprise qui a élaboré le système de classification des emplois qui inspire l'accord de la métallurgie de 1975. Tous ces exemples sont donnés pour tenter de vous convaincre de vous poser la question de l'apport des pratiques de management à la mise en œuvre de la démocratie industrielle: quelle place donner aux entreprises dans le processus d'institutionnalisation et la démocratie industrielle?

Le rapport de Jean Saglio et de Sophie le Corre sur le grand commerce alimentaire montre une relation de l'État et des entreprises du secteur à travers l'écriture du droit. Il montre que l'écriture du droit est, au début de la période étudiée, une formalisation des usages professionnels. Le ministère du Travail a une position d'arbitre entre le patronat et les syndicats, mais il intervient aussi pour formaliser les usages. Cette intervention est tellement importante et vitale pour les entreprises que le patronat reproche au ministère de ne pas aller assez vite. Il lui demande aussi de rédiger des textes suffisament clairs.

Ma première question porte sur la chronologie. Le rapport présente une chronologie institutionnelle mais compte tenu du caractère local du secteur, ne seraitil pas utile de prendre aussi en compte une chronologie de l'urbanisme commercial? La législation qui permet la création de grands magasins a peut-être un rapport avec l'évolution du droit social et les possibilités de négociations. Ma seconde question a trait au politique. Je me demande pourquoi le politique est très peu présent (je l'ai repéré pour la loi de 1936 où l'on voit que le secteur de l'épicerie ne respecte pas la durée légale du travail mais qu'au fond, ce n'est pas très grave parce qu'il a des soutiens politiques qui lui permettent de continuer à faire comme d'habitude). Il me semble que la politique, dans ce secteur-là, est pourtant très importante. C'est même un rapport très conflictuel si l'on pense, par exemple, à l'action de Pierre Poujade. Le rapport au politique est particulièrement complexe dans ce secteur d'activité et c'est un point que le rapport donne envie d'approfondir.

\section{- Michèle Tallard}

Nous avons mobilisé pour l'essentiel des sources d'archives. Pour la France, les fonds d'archives de la direction des relations du Travail, les fonds d'archives syndicaux et des témoignages, notamment avec le groupe protagoniste de la commission Sudreau; pour l'Allemagne, on a utilisé toutes les revues syndicales complétées par le fonds Schiller 
qui vient d'ailleurs d'être versé aux archives fédérales à Coblence.

\section{- Catherine Vincent}

Sur les positions syndicales, ce qu'il y a de commun en France au-delà des divergences syndicales c'est un très grand malaise par rapport à cette question de la démocratie industrielle et puis tout particulièrement du pouvoir dans l'entreprise: estce qu'on doit le partager? Est-ce qu'on doit intervenir dans les stratégies de l'entreprise? Il faut mettre à part la CGC qui avait une position assez ouverte et certainement proche de celles des syndicats allemands. D'ailleurs, dans les années 1970 la seule traduction législative qui pouvait ressembler à la codétermination, c'est la participation des cadres au conseil d'administration: c'est une loi de 1972 portée par la CGC et qu'elle arrive à faire passer, à ce moment-là, avec l'opportunité de l'arrivée de Jacques Chaban-Delmas et de son projet de nouvelle société.

Les autres syndicats sont mal à l'aise vis-à-vis de cette question, même s'ils ont des positions parfois divergentes. Il y a à cet égard une continuité entre ce qu'est la CFTC au début des années 1960 et la CFDT des années 1970, puisque les courants majoritaires qui réfléchissent au sein de la CFTC sur la démocratie industrielle vont fonder la CFDT. La CFDT est tentée par une participation, ce qui lui vient de son origine CFTC tout en ayant une appréciation assez négative de la codétermination allemande qui est perçue comme une collaboration de classes. Avant la diffusion du terme «d'autogestion», il existe une volonté d'affirmer un pouvoir d'influence, de contrôle autonome des travailleurs face à la stratégie de l'entreprise. La position de la CGT est peut-être un peu plus simple: elle est très négative vis-à-vis de la réflexion sur le pouvoir dans l'entreprise. Elle souhaite affirmer la présence syndicale dans l'entreprise et développer la formation et la consultation autour du comité d'entreprise. Point commun avec la CFDT, elle attend un changement politique, avec la signature du programme commun dans lequel la CGT s'investit beaucoup.

\section{- Michèle Dupré}

La situation syndicale est plus simple en Allemagne. Par exemple, les syndicats d'employés jouent un rôle très important. Ceci étant, d'après les archives du ministère, le partenaire majeur du ministère du Travail, quel que soit le gouvernement en place, est constitué par les grandes fédérations. Nous avons utilisé volontairement dans le rapport le mot codétermination alors qu'à l'époque (notamment chez les syndicats français), on utilise plus volontiers le mot cogestion. C'est pour bien montrer comment les discours s'intègrent dans une histoire croisée à partir de concepts élaborés dans d'autres pays mais utilisés pour développer leurs les propres stratégies au niveau national. Le patronat français, s'il est favorable à la loi sur les comités d'entreprises, ne veut pas entendre parler d'une évolution vers la participation au conseil de surveillance. On trouve aussi dans les archives du ministère allemand des réactions du CNPF: le ministère et le patronat allemand cherchent à tirer argument du positionnement du patronat français.

\section{- Sophie le Corre}

Est-ce que le commerce alimentaire a une longue tradition de résistance à la réglementation? Qu'estce que le secteur du commerce alimentaire et du grand commerce alimentaire? Ces questions sont complexes, car les réponses changent selon les périodes. La tradition de résistance à la réglementation est à la fois vraie et fausse. Elle est vraisemblablement moins du côté patronal qu'on ne le pense spontanément. Les travaux par exemple de Jean-Pierre Hirsch sur les entrepreneurs négociants du Nord de la France montrent que les négociants ne résistent pas tellement à la réglementation parce qu'ils appliquent une réglementation qui leur convient. S'agissant du commerce, c'est d'autant plus important qu'on est dans un secteur de maind'œuvre, où la double question du salaire et de la rémunération du travail est fondamentale. La production se fait sous condition de durée d'ouverture des boutiques, question non réglementée en France et qui ne l'est qu'à travers la question de la durée du travail. Définir le périmètre du commerce est complexe car les choses changent tout le temps, en particulier à partir des années 1950 en France. Du côté des syndicats de salariés, il y a des dossiers sur lesquels ils auraient aimé l'arbitrage du ministère du Travail qu'ils ont d'ailleurs obtenu en 1937 mais pas complètement. C'est le dossier, en particulier, de la durée du travail avec la fameuse question des heures d'équivalence entre heures de présence au travail et durée effective du travail. Ces décrets n'ont pas toujours été abrogés, ils ont toujours cours. La seule solution qui a été trouvée dans les secteurs du commerce est une solution conventionnelle et non pas réglementaire avec extension par le ministère du Travail. L'équivalence est fixée de la façon suivante: 46 heures de présence valent et sont payées 40 heures, durée fixée par la loi de 1936. Il y a d'autres exemples comme la question du travail du dimanche. Un autre exemple concerne la fusion des deux conventions collectives nationales à la fin des années 1990. Le ministère du Travail tarde très longtemps à étendre cette convention. Il y a un écart entre la réception de ces deux conventions qui ont fonctionné en parallèle de 1969 jusqu'à la fin des années 1990 au moment où elles sont fusionnées par les partenaires sociaux de la branche. Elles étaient jumelles, sauf en ce qui concerne les classifications, bien sûr, puisque d'un côté on avait les entrepôts et de l'autre les magasins. Du point de vue des partenaires sociaux, il n'y avait aucune difficulté, si ce n'est technique, à les fusionner, ni à obtenir l'extension. 


\section{- Bruno Guérard, inspecteur du Travail honoraire}

Je souhaite souligner la richesse des débats de cette dernière séance qui nous montrent la difficulté à résumer une histoire d'une durée, pour les conventions collectives, d'à peu près 90 ans (ou pour les institutions sociales de l'entreprise, d'un peu plus de 50 ans). Par exemple, sait-on vraiment si les comités d'entreprise étaient en baisse de potentiel dans les années 1965 ou 1970, ou si ce n'est pas précisément l'inverse? Je pense aussi qu'il est difficile d'étudier des mots ou la circulation de concepts généraux sur une longue période, l'institution perdure avec un autre sens et les mots ne recouvrent plus les mêmes réalités. Cette séance me semble ouvrir un chantier où il n'est pas facile de faire le rapprochement entre les différentes archives. En matière de conventions collectives, nous avons eu une présentation du rôle du ministère du Travail à partir du grand commerce alimentaire, mais ce n'est peut-être pas l'élément le plus marquant pour décrire l'attitude du ministère du Travail face au système conventionnel par rapport à des secteurs comme la métallurgie, la chimie ou le textile. Ce chantier reste à mon avis complètement ouvert, car il est prématuré de tirer des principes ou des notions générales sur la culture sociale du ministère du Travail à partir d'une réalité extrêmement riche et qui subit des fluctuations sur plusieurs décennies, de surcroît en liaison, vous nous l'avez montré, avec les réalités des pays étrangers. 